\title{
TetI in Nucleus Accumbens Opposes Depression- and Anxiety-Like Behaviors
}

\author{
Jian Feng',2, Catherine J Pena', Immanuel Purushothaman', Olivia Engmann', Deena Walker', \\ Amber N Brown'2, Orna Issler', Marie Doyle', Eileen Harrigan', Ezekiell Mouzon', Vincent Vialou', Li Shen', \\ Meelad M Dawlaty ${ }^{3}$, Rudolf Jaenisch ${ }^{4,5}$ and Eric J Nestler, \\ 'Fishberg Department of Neuroscience and Friedman Brain Institute, Icahn School of Medicine at Mount Sinai, New York, NY, USA; ${ }^{2}$ Department \\ of Biological Science, Florida State University, Tallahassee, FL, USA; ${ }^{3}$ Department of Genetics and Gottesman Institute for Stem Cell and \\ Regenerative Medicine Research, Albert Einstein College of Medicine, Bronx, NY, USA; ${ }^{4}$ Whitehead Institute for Biomedical Research, Cambridge, \\ MA, USA; ${ }^{5}$ Department of Biology, Massachusetts Institute of Technology, Cambridge, MA, USA
}

\begin{abstract}
Depression is a leading cause of disease burden, yet current therapies fully treat $<50 \%$ of affected individuals. Increasing evidence implicates epigenetic mechanisms in depression and antidepressant action. Here we examined a possible role for the DNA dioxygenase, ten-eleven translocation protein I (TETI), in depression-related behavioral abnormalities. We applied chronic social defeat stress, an ethologically validated mouse model of depression-like behaviors, and examined Tet I expression changes in nucleus accumbens (NAc), a key brain reward region. We show decreased Tetl expression in NAc in stress-susceptible mice only. Surprisingly, selective knockout of Tetl in NAc neurons of adult mice produced antidepressant-like effects in several behavioral assays. To identify Tet I targets that mediate these actions, we performed RNAseq on NAc after conditional deletion of Tet I and found that immune-related genes are the most highly dysregulated. Moreover, many of these genes are also upregulated in the NAc of resilient mice after chronic social defeat stress. These findings reveal a novel role for TETI, an enzyme important for DNA hydroxymethylation, in the brain's reward circuitry in modulating stress responses in mice. We also identify a subset of genes that are regulated by TETI in this circuitry. These findings provide new insight into the pathophysiology of depression, which can aid in future antidepressant drug discovery efforts.

Neuropsychopharmacology (2017) 42, I657-1669; doi:I0.1038/npp.2017.6; published online 25 January 2017
\end{abstract}

\section{INTRODUCTION}

Depression is a recurring and life-threatening illness that affects up to $20 \%$ of the population, yet $<50 \%$ of patients respond fully to available treatments, which highlights the need for a better understanding of the syndrome and for improved treatments (Hyman, 2014; Krishnan and Nestler, 2008). Epigenetic mechanisms can encode environmental stimuli into behavioral adaptations throughout an individual's lifetime and have been implicated increasingly in several neuropsychiatric disorders, including depression (Akbarian, 2014; Bagot et al, 2014; Vialou et al, 2013). DNA methylation is a key epigenetic mechanism where methyl groups are covalently coupled to the C5 position of cytosine (5-methylcytosine $(5 \mathrm{mC})$ ) (Jaenisch and Bird, 2003). The epigenetic modification of DNA provides an attractive regulatory mechanism underpinning the transcriptional alterations that contribute to the behavioral

\footnotetext{
*Correspondence: Dr EJ Nestler, Fishberg Department of Neuroscience and Friedman Brain Institute, Icahn School of Medicine at Mount Sinai, One Gustave L. Levy Place, Box 1065, New York, NY |0029-6574, USA, Tel: +I 212659 5656, Fax: +I 2126591559. E-mail: eric.nestler@mssm.edu

Received 16 September 2016; revised 3 January 2017; accepted 5 January 2017; accepted article preview online II January 2017
}

abnormalities in brain disorders. However, the existence of a DNA methylation turnover pathway in the brain and its potential role in neural disorders have been elusive.

Ten-eleven translocation protein 1 (TET1) oxidizes $5 \mathrm{mC}$ into 5hydroxymethylcytosine $(5 \mathrm{hmC})$ (Kriaucionis and Heintz, 2009; Tahiliani et al, 2009). TET1, and the related family members TET2 and TET3, can also further oxidize $5 \mathrm{hmC}$, eventually leading to unmethylated cytosine. This provides a mechanism by which $5 \mathrm{mC}$ oxidation mediates active DNA demethylation in the brain (Cheng et al, 2015; Guo et al, 2011). Although $5 \mathrm{hmC}$ is most enriched in the brain, the involvement of TETs and $5 \mathrm{hmC}$ in the regulation of adult brain function remains poorly understood. TETs and $5 \mathrm{hmC}$ have been shown to mediate active DNA demethylation in the hippocampus where they influence neural development, aging, neural plasticity, and learning and memory (Guo et al, 2011; Kaas et al, 2013; Li et al, 2014; Rudenko et al, 2013; Szulwach et al, 2011; Yu et al, 2015; Zhang et al, 2013). Recent evidence also suggests the involvement of TET/5hmC in neuropsychiatric disorders (Feng et al, 2015; Guidotti et al, 2013). For example, we found that TET1, acting in mouse nucleus accumbens (NAc) - a key reward region-negatively regulates cocaine reward behavior through widespread dynamic changes of $5 \mathrm{hmC}$ at responsive genes (Feng et al, 2015). In this study, 
we explored a potential role of TET1 in stress responses after chronic social defeat stress (CSDS), an ethologically validated model of depressive-like behaviors (Berton et al, 2006; Dias et al, 2014; Golden et al, 2011; Krishnan et al, 2007).

\section{MATERIALS AND METHODS}

\section{Animals}

For CSDS, 7-9-week-old male c57bl/6 mice from Jackson Laboratories were used. All mice were housed on a 12-h light/dark cycle with ad libitum access to food and water. CD1 retired breeder male mice were obtained from Charles River Laboratories. Tet $1^{\text {loxP/loxP }}$ mice (Dawlaty et al, 2011; Zhao et al, 2015) were backcrossed to a c57bl/6 background. Male homozygous offspring aged 7-9 weeks were used for viral manipulations and behavior assays. Biochemical assays were performed on bilateral 14 gauge punches of NAc. The Mount Sinai IACUC approved all experimental protocols.

\section{Chronic Social Defeat Stress}

CSDS was performed as described (Dias et al, 2014; Golden et al, 2011). Briefly, an episode of social defeat involves placing a test intruder mouse into the home cage of a prescreened CD1 aggressor mouse, leading to an agonistic encounter. After $10 \mathrm{~min}$, mice are separated by a perforated divider for the remainder of the 24 -h period. This process is repeated daily for 10 days, each day with a novel CD1 mouse. In parallel, control animals are placed in pairs within an identical home cage setup, one control animal per side divided by a perforated divider, for the duration of the defeat sessions. After the last defeat session on day 10, all intruder and control mice are singly housed. Behavioral testing (eg, social interaction (SI)) was performed $24 \mathrm{~h}$ after the last defeat. A subgroup of defeated animals (termed 'susceptible') demonstrate marked social avoidance, which is associated with other behavioral and physiological changes reminiscent of depressive and anxiety symptoms. Social defeat also produces a subgroup of animals (termed 'resilient') that fails to develop social avoidance. Prior research has shown that susceptibility $v s$ resilience is not related to the severity of aggression or injuries sustained (Krishnan et al, 2007). Bilateral 14 gauge NAc punches are collected $48 \mathrm{~h}$ after the last defeat unless otherwise noted.

\section{RNA Isolation and qPCR}

RNA was extracted and purified using a Trizol-based protocol (Feng et al, 2015), as measured on a Nanodrop spectrophotometer. RNA was then reverse transcribed into cDNA with the iScript DNA Synthesis Kit (Bio-Rad). Realtime qPCR was performed with the $\Delta \Delta \mathrm{Ct}$ method to obtain relative fold change of expression as compared with control samples. GAPDH was utilized for normalization. Primers used in this study include:

Anxa2: 5'-CATCTGCTCACGAACCAACC-3', 5'-TCAG CTTTCGGAAGTCTCCAG-3';

Bst2: 5'-CTGTAGAGACGGGTTGCGA-3', 5'-CTTCTTC TCCAGGGACTCCTGA-3';

Cd74: 5'-TCCCAGAACCTGCAACTGGA-3', 5' -ATCAGCAAGGGAGTAGCCATC-3';
Fgl2: 5'-CCAGCCAAGAACACATGCAG-3', 5'-GGGTA ACTCTGTAGGCCCCA-3';

Gbp6: 5'-ACTGAGAAGGAAGCTGGAGCAG-3', 5'-TCT

CTCAGTTGCTGTATCTCTTTGT-3';

H2-Aa: 5'-GACCTCCCAGAGACCAGGAT-3', 5' -ACCA-

TAGGTGCCTACGTGGT-3';

H2-Ab1: 5'-TTAGGAATGGGGACTGGACCT-3' ', 5' -TCT

TGCTCCAGGCAGACTCA-3';

H2-Eb1: 5'-TCCGAAATGGAGACTGGACC-3', 5'-TGTT

CTGTGCAGATGTGGATTG-3';

Iigp1: 5'-GGGGTGGGTCTCATGTGAAG-3', 5'-CCAAT-

CACAGGCAAGTGTGC-3';

Lcp2: 5'-TGACTATGAGCCTCCACCCTC-3'， 5'-TTTG GTCTCAGTGGGGGCAC-3';

Lyz2: 5'-TGCTCAGGCCAAGGTCTATG-3'， 5'-TGGTC TCCACGGTTGTAGTT-3';

Ngb: 5'-AGGACTGTCTCTCCTCTCCAG-3', 5'-CAAGC TGGTCAGGTACTCCTC-3';

Plscr1: 5'-TGTGTAGCTGCTGTTCCGAC-3', 5'-ACATCCAGGTCTAGCGGGAA-3';

Serping1: 5'-AGTGCCCATGATGAGTAGCG-3', 5'-CAC GGGTACCACGATCACAA-3';

Slc12a7: 5'-CAACAAGCTGGCACTGGTCT-3', 5' -TCAA

AGTTGCGATTTGCCAGC-3';

Cxcl13: 5'-ATTCAAGTTACGCCCCCTGG-3', 5'-TTGG

CACGAGGATTCACACA-3';

Cxcl9: 5'-CGAGGCACGATCCACTACAA-3', 5'-CTTCACATTTGCCGAGTCCG-3';

Ighg1: 5'-ACAGCACTTTCCGTTCAGTCA-3', 5'-GTGTA

CACCTGTGGAGCCTTC-3';

Igkv6-23: 5' -CATGGGCATCAAGATGGAGAC-3', 5' -CA-

CATCCTGACTGGCCTTGC-3';

Tet1: 5'-GTCAGGGAGCTCATGGAGAC-3', 5'-CCTGAGAGCTCTTCCCTTCC-3';

Tet2: 5'-GCAAGAGCTCTCAGGGATGT-3'， 5'-AGGTC GCACTCGTACCAAAC-3';

Tet3: 5'-CCAAGGCAAAGACCCTAACA-3', 5' -AGCAAC TTCAGTGGCCAGAT-3';

Tet1 exon 4: 5'-AGGTACACAAAAAGAAAAAGGCC C-3', 5'-CCATGAGCTCCCTGACAGC-3';

Tet1 exons 4 and 5 (Dawlaty et al, 2011): 5'-GTCAGGGAGCTCATGGAGAC-3', 5'-CCTGAGAGCTCTTCCCTT $\mathrm{CC}-3^{\prime}$ and

GAPDH: 5'-GGGTGTGAACCACGAGAAAT-3', 5'-GTC TTCTGGGTGGCAGTGAT-3'.

\section{Stereotaxic Surgeries}

Surgery was performed under ketamine/xylazine anesthesia. AAV-Cre or AAV-GFP was infused bilaterally into the NAC at a rate of $0.1 \mu \mathrm{l} / \mathrm{min}$ with the following coordinates: $+1.6 \mathrm{~mm} \mathrm{~A} / \mathrm{P},+1.5 \mathrm{~mm} \mathrm{M} / \mathrm{L}, \pm 4.4 \mathrm{~mm} \mathrm{D} / \mathrm{V}$ from Bregma. A total of $0.5 \mu \mathrm{l} /$ side was infused. All vectors were purchased from UNC Viral Core Facility. Behavioral assays were performed 4 weeks after viral injection. At the end of experiments, the brains of all animals were studied to confirm the accuracy of viral injections.

\section{Behavioral Tests}

Mice subjected to CSDS or control conditions were examined in a battery of tests in the following order: SI, 
sucrose preference, open field, and elevated-plus maze (EPM).

Social interaction. An SI test, performed as described (Golden et al, 2011), evaluates an experimental mouse's interaction with an empty cage $v s$ a cage containing a novel CD1 target mouse. This test is used to distinguish susceptible mice, those that show decreased SI after CSDS, from resilient mice, those that avoid this abnormality (Berton et al, 2006; Krishnan et al, 2007). Briefly, an experimental mouse was allowed to explore an arena with an empty wire holding chamber for $150 \mathrm{~s}$, immediately followed by $150 \mathrm{~s}$ of exploration in the same arena with a novel CD1 target mouse within the holding chamber. Ethovision software (Noldus) tracked animal movement from live video. Less time spent investigating the 'interaction zone' immediately surrounding the holding chamber containing the social target has been validated as depressive-like susceptible behavior.

Sucrose preference. Individually housed mice were first habituated to two bottles of water for 1 day, followed by 3 consecutive days with one bottle each of water and $1 \%$ sucrose (Krishnan et al, 2007). Consumption was measured by daily weighing, after which the two bottles were switched.

Open field. Each mouse is placed in the center of a chamber which they freely explore (Krishnan et al, 2007), with their activity and location measured by videotracking. Mice are allowed to freely explore the chamber, and they will typically spend a significantly greater amount of time exploring the periphery of the arena, usually in contact with the walls, than the unprotected center area. Mice that spend significantly more time exploring the unprotected center area demonstrate anxiolytic-like baseline behavior.

Elevated-plus maze. Mice are placed in the center of an EPM, consisting of two interleaved open and closed arms, elevated 4 feet off the ground (Krishnan et al, 2007). Animals were placed in the center and time spent in open $v s$ closed arms is measured using the Ethovision tracking software for $5 \mathrm{~min}$. Measurement is of the time spent in the open arm or closed arm of maze.

\section{RNA Sequencing (RNAseq)}

RNA integrity was confirmed by Bioanalyzer with RIN $>8.0$. In all, $0.5 \mu \mathrm{g}$ of total RNA was used for library construction using the Illumina Truseq mRNA Sample Prep Kit. All sequencing data were processed as previously described (Feng et al, 2014), with the voom package (Law et al, 2014) used for differential analyses with a cutoff of $30 \%$ change ( $>1.3$-fold or $<0.7$-fold) and $P$-value $<0.05$. Gene ontology analyses were carried out by DAVID (Huang da et al, 2009) with highest stringency settings. All RNAseq data are deposited into the Gene Expression Omnibus with accession number GSE76977.

\section{Statistical Analysis}

Prism statistics package was used for data analyses. Twotailed Student's $t$-test were used with statistical significance at

\section{b}
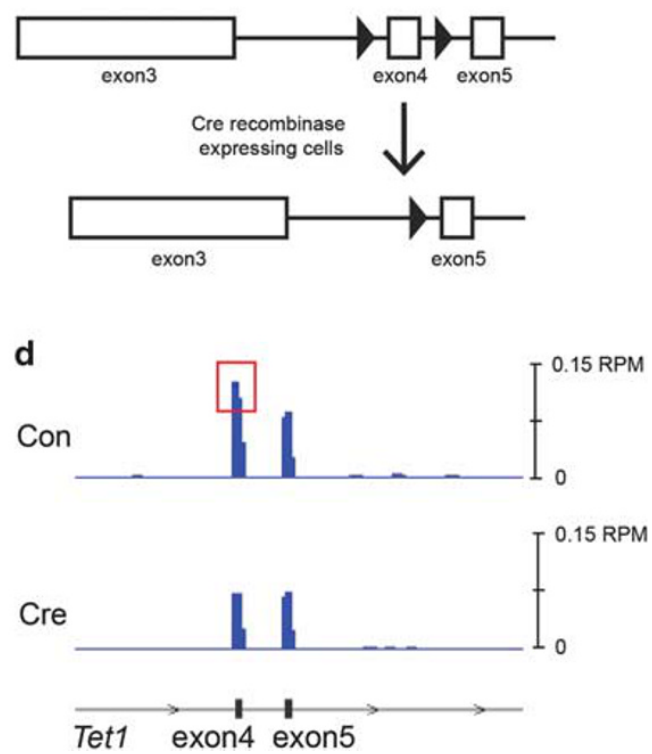

Figure I Regulation of Tet l expression by CSDS. (a) Tet I mRNA levels are decreased in the NAc of susceptible mice 48 h after CSDS. (Con: control, Sus: susceptible, Res: resilient. $N=8$ for each group. Two-tailed $t$-test, $*$ P $=0.025$.) (b) Schematic of floxed Tet I locus (Dawlaty et al, 20I I). Open boxes indicate exons of Tetl gene, black triangles represent loxP sites flanking exon 4, which is excised in the presence of Cre. (c) qPCR validation of Tetl decrease after AAV-Cre injection in NAc of floxed Tet I mice (Cre) as compared with AAV-GFP controls (Con). Two primer sets were used, which cover exon 4 alone (Tet l primer set I) or both exons 4 and 5 (Tet l primer set 2) ( $N=7$ for each group. Two-tailed $t$-test, $* P=0.01$ I, *** $P=0.005$ ). (d) RNAseq read counts of Tet I. Schematic of relative position of exons 4 and 5 is shown on the bottom. Blue traces represent normalized read counts across these three exons in both control (Con) and Cre conditions under the same scale. Red box highlights differential reading of exon 4. 

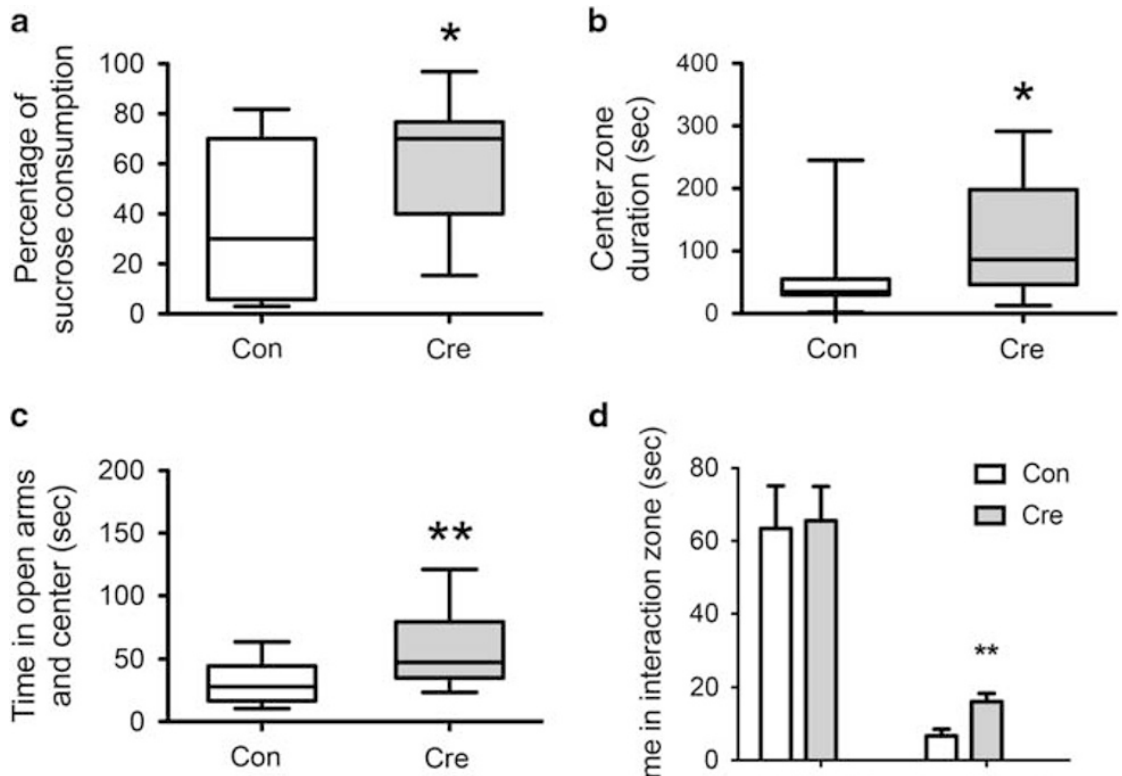

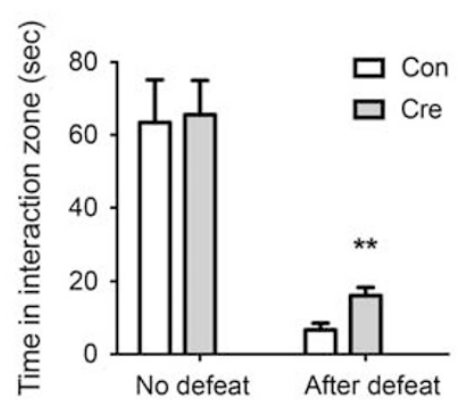

e Social Avoidance Susceptibility

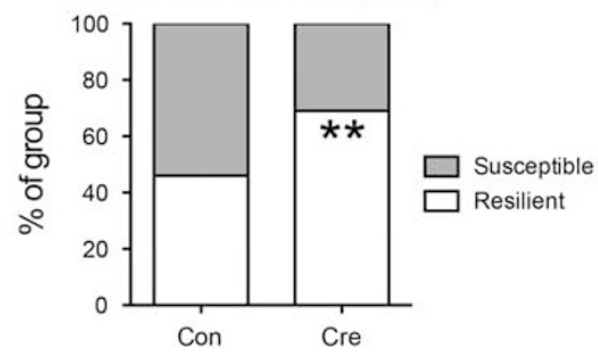

Figure 2 Tet/ KO from NAc induces antidepressant- and anxiolytic-like effects. (a) Floxed Tet I mice that received AAV-Cre in NAc exhibit increased sucrose preference $(N=15$ for each group. Two-tailed $t$-test, $* P=0.023)$. (b) Cre mice also spent more time in the center zone in the open filed test $(N=15$ for each group. Two-tailed $t$-test, $* P=0.024)$ and $(c)$ spent more time in the open arms in the EPM $(N=15$ for each group. Two-tailed $t$-test, $* * P=0.008)$. (d) Cre mice exhibited a partial rescue of social avoidance after $\operatorname{CSDS}(N=13$ for each group. Two-tailed $t$-test, $* * *=0.004)$. (e) Cre mice demonstrated a greater percentage (** $P=0.00$ I by a chi-squared test) of resilience ( $69 \%$ resilient vs $31 \%$ susceptible) than control mice ( $46 \%$ resilient vs $54 \%$ susceptible) I month after CSDS.

$P<0.05$. A chi-squared test was carried out to analyze the percentage of resilient mice after Tet1 deletion.

\section{RESULTS}

We first tested whether Tet1 expression in NAc is affected by CSDS. In CSDS, $\sim 65 \%$ of defeated animals (termed 'susceptible') demonstrate key behavioral abnormalities, such as social avoidance, with the remaining $\sim 35 \%$ (termed 'resilient') not presenting these symptoms (Krishnan et al, 2007). We focused our studies on NAc based on its role in reward and motivation and its implication in the anhedonic aspects of depression (Russo and Nestler, 2013). Examining NAc tissue $48 \mathrm{~h}$ after the last defeat, we found a selective decrease in Tet1 mRNA levels in the NAc of susceptible mice, but not in resilient mice, as compared with undefeated controls (Figure 1a). In contrast, neither Tet 2 nor Tet3 expression was changed in either susceptible or resilient mice.

To study the functional consequences of Tet 1 suppression in NAc of susceptible mice, we injected AAV-Cre or AAV-
GFP bilaterally into NAc of Tet $1^{\text {loxP/loxP }}$ mice (Dawlaty et al, 2011), in which exon 4 is flanked by loxP sites (Figure 1b). By using two independent Tet1 primer sets targeting exon 4, we confirmed a small but significant decrease of Tet 1 transcripts in NAc 4 weeks after AAV-Cre injection (Figure 1c). The magnitude of decrease $(\sim 20 \%)$ is consistent with previous viral-mediated knockdowns (Dias et al, 2014) and likely reflects the fact that the AAV vectors used infected neurons only and that microdissections unavoidably contain noninfected tissue. The Tet1 knockout (KO) was further confirmed by our RNAseq data (see below), where normalized read counts of Tet 1 exon 4 were similarly decreased in AAV-Cre- $v s$ AAV-GFP-treated animals (Figure 1d).

We next studied mice with a Tet $1 \mathrm{KO}$ in NAc in a battery of baseline behavioral assays. Tet1 NAc-KO mice displayed increased preference of sucrose (Figure 2a). This result suggests that reduced Tet1 expression in this brain region produces an antidepressant-like effect. Tet1 NAc-KO mice also spent more time in the center zone in the open field test (Figure 2b), indicating a decrease in anxiety-like behavior. Further evidence for an anti-anxiety-like effect is increased 
Table I List of Differential Genes After Tet I KO in Mouse NAc

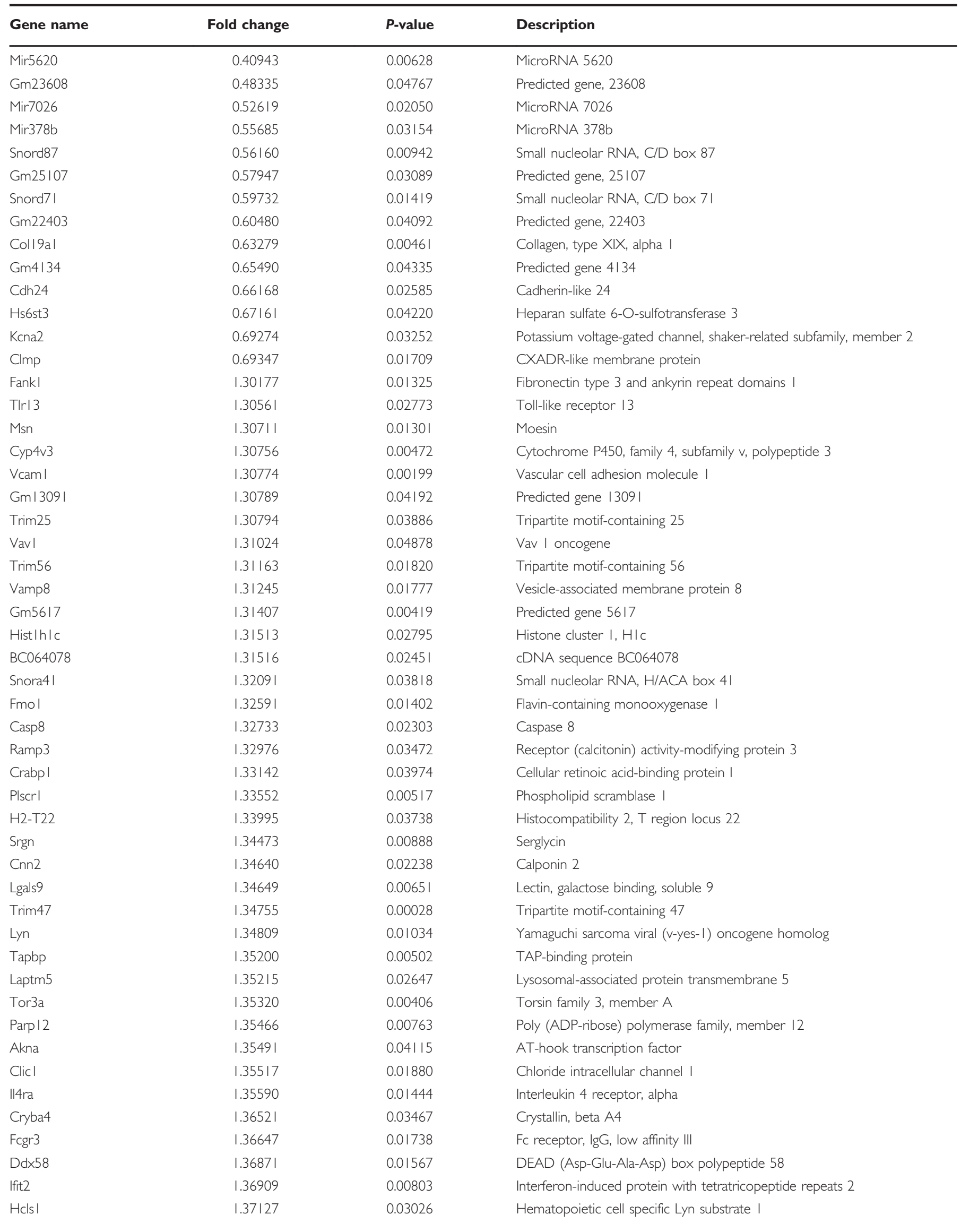


Table I Continued

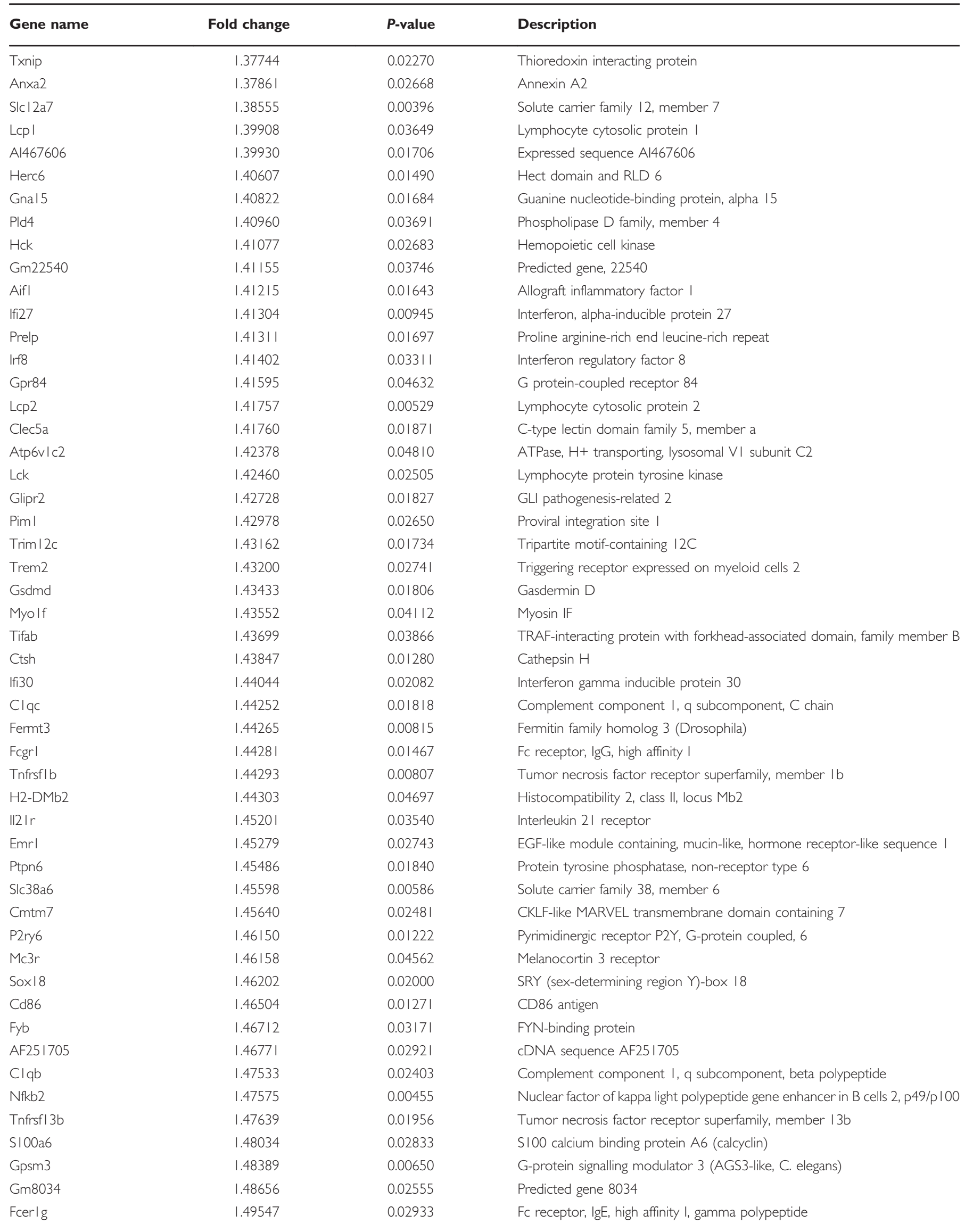


Table I Continued

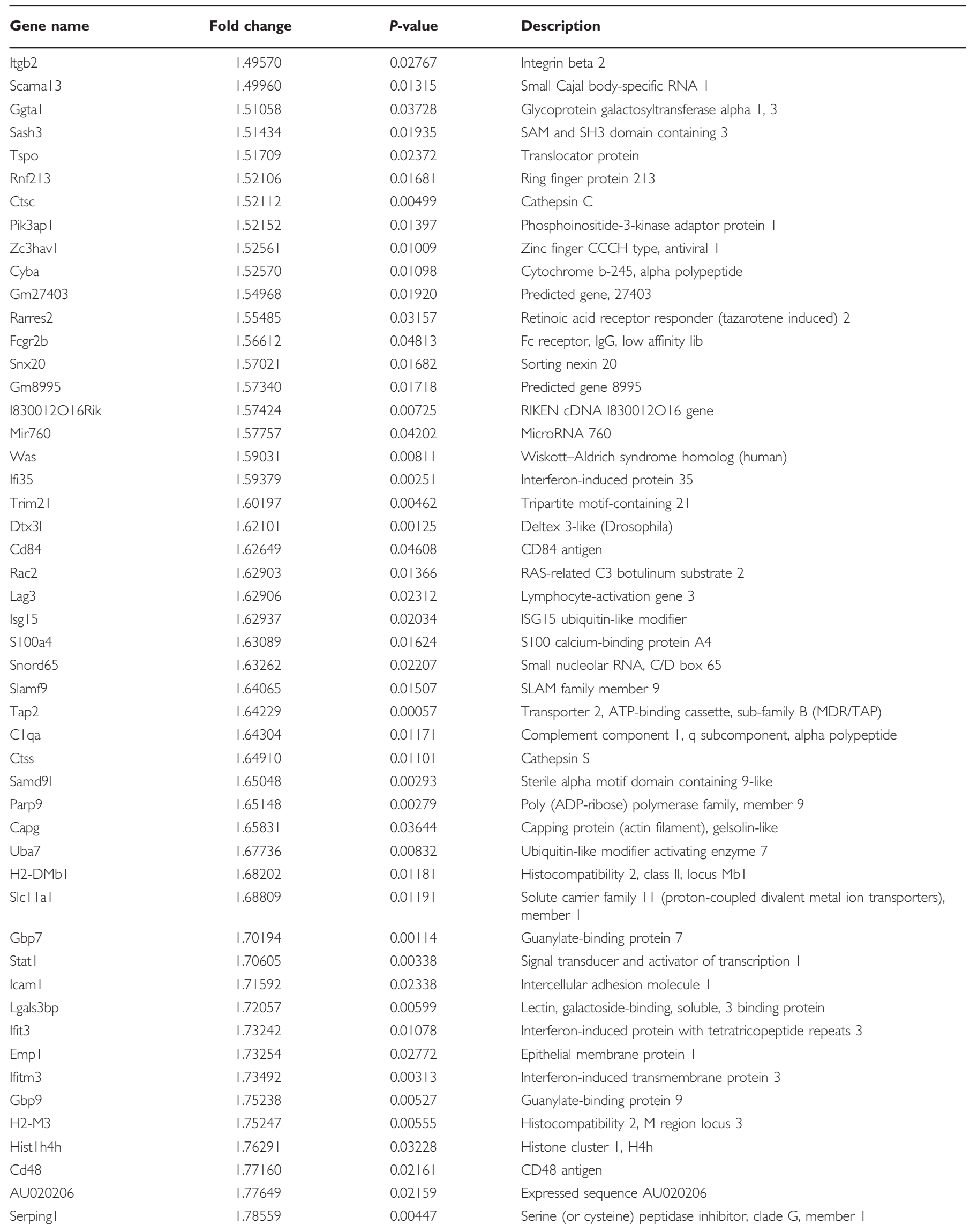


Table I Continued

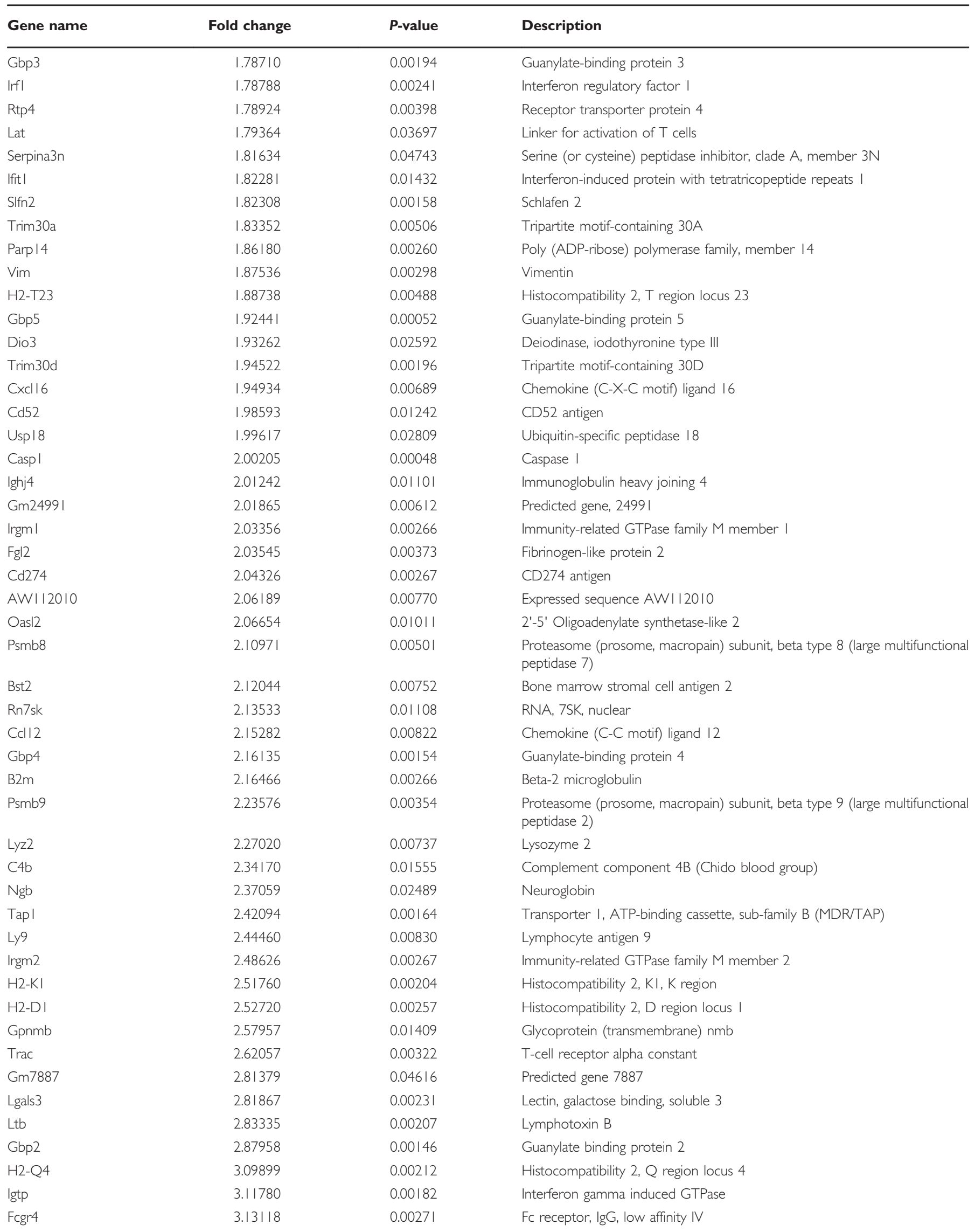


Table I Continued

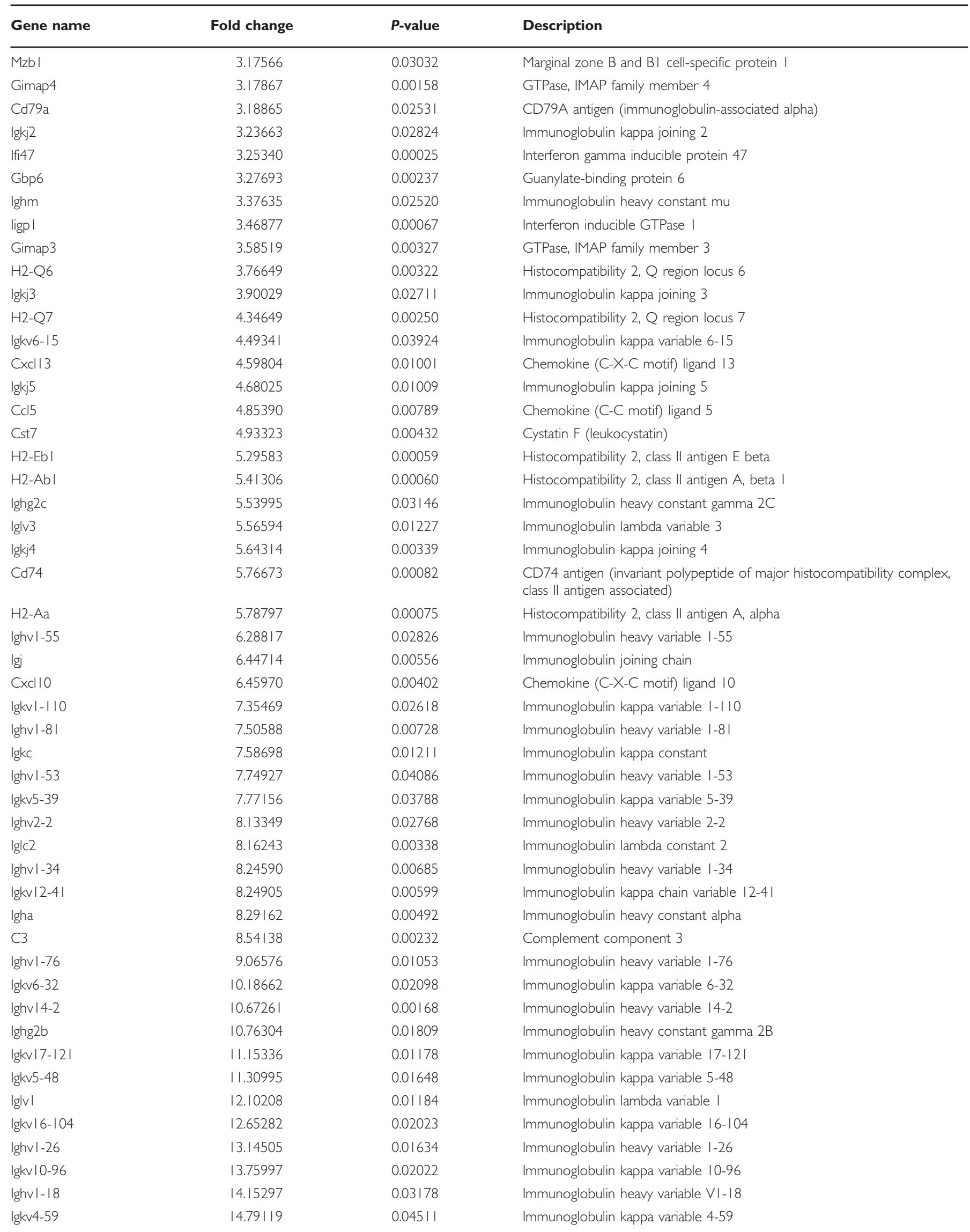


Table I Continued

\begin{tabular}{|c|c|c|c|}
\hline Gene name & Fold change & $P$-value & Description \\
\hline $\mathrm{CxCl9}$ & $|4.8053|$ & 0.00085 & Chemokine (C-X-C motif) ligand 9 \\
\hline |ghv|-82 & | 4.81065 & 0.00222 & Immunoglobulin heavy variable |-82 \\
\hline lgkv8-28 & | 5.05572 & 0.00206 & Immunoglobulin kappa variable 8-28 \\
\hline |ghv|-64 & | 5.63836 & 0.00836 & Immunoglobulin heavy variable |-64 \\
\hline |ghvl-52 & 15.87288 & 0.02147 & Immunoglobulin heavy variable |-52 \\
\hline Igkv6-I7 & $|8.033|$ | & 0.00177 & Immunoglobulin kappa variable 6-I7 \\
\hline Ighv9-3 & 21.83215 & 0.00297 & Immunoglobulin heavy variable V9-3 \\
\hline$|g k v| 2-46$ & $25.9777 \mid$ & 0.01001 & Immunoglobulin kappa variable I2-46 \\
\hline Igkv9-120 & 26.31750 & 0.00110 & Immunoglobulin kappa chain variable 9-120 \\
\hline
\end{tabular}

time in the open arms of the EPM (Figure 2c). Of note, we did not detect significant changes in SI or forced swimming in non-stressed animals. Exposing Tet1 NAc-KO and control mice to CSDS revealed that, although both groups showed social avoidance behavior, the Tet1 NAc-KO mice displayed a partial reversal of this deficit (Figure 2d). Tet1 NAc-KO also increased the percentage of resilient animals after CSDS (Figure 2e). This finding further supports an antidepressantlike action upon Tet1 KO. Importantly, previous work has shown that overexpression of Cre in NAc of wild-type mice has no effect on any of these behavioral end points (Dias et al, 2014; Krishnan et al, 2007).

To understand the molecular underpinnings of these behavioral effects, we performed RNAseq to examine the gene expression changes in NAc upon a local Tet1 $\mathrm{KO}$ without stress experience. The predominant effect of loss of Tet 1 is gene induction, with 252 genes upregulated in Tet 1 NAc-KO and only 14 genes downregulated (Table 1). Gene ontology analysis revealed that a large majority of the upregulated genes are concentrated in immune-related categories (Figure 3a). We then overlaid the upregulated and downregulated gene lists upon Tet1 NAc-KO with an RNAseq data set of CSDS-induced gene expression changes in NAc 4 weeks after the last defeat (Bagot et al, 2016). This data set identified 140 upregulated and 86 downregulated genes in the resilient subgroup, and the upregulated genes showed a significant overlap with genes upregulated upon Tet1 KO ( $N=15$ genes, $P<1.586 \mathrm{e}-09$, Figure $3 \mathrm{~b}$ and $\mathrm{d})$. In contrast, only four upregulated genes in Tet $1 \mathrm{KO}$ were also induced in NAc of susceptible mice $(P<0.022$, Figure $3 c)$.

To further confirm the potential molecular targets regulated by Tet $1 \mathrm{KO}$, we carried out quantitative PCR analyses on a set of 15 genes that demonstrate induction in NAc upon local Tet1 KO based on our RNAseq data. Indeed, the majority $(N=11)$ of them were confirmed to have a significant increase after Tet1 deletion (Figure 4), with most of the rest showing a trend toward increasing as well.

\section{DISCUSSION}

We found a selective decrease in Tet1 expression in NAc of mice that are susceptible to CSDS, an effect not seen in resilient mice. By use of viral-Cre-mediated deletion of Tet 1 in NAc neurons of adult mice, we showed that loss of Tet 1 in this brain region mediates antidepressant- and anxiolytic-like effects and that these behavioral actions are associated with the predominant induction of immune-related genes upon Tet1 NAc-KO. The finding of a molecular change (Tet1 suppression) in susceptible mice, but not in resilient mice that opposes the behavioral abnormalities associated with susceptibility, is surprising. It raises the interesting hypothesis that Tet1 suppression in NAc is a homeostatic adaptation to counter susceptibility, which is not necessary in resilient mice that achieve resilience through other mechanisms. Indeed, the finding that a subset of genes induced in NAc upon Tet1 NAc-KO are also induced in resilient (but not susceptible) mice supports this interpretation. The observation that Tet 1 NAc-KO only partially rescues the deleterious effects of CSDS supports the known involvement of many other genes in stress susceptibility (eg, Berton et al, 2006; Krishnan et al, 2007; Sun et al., 2015). In the future, it will be important to validate these findings in other stress models.

Increasing evidence supports a role for DNA methylation in mediating the effects of stress on the brain (Bagot et al, 2014). One well-studied example is glucocorticoid receptor (GR) gene methylation in response to early-life conditions (Turecki and Meaney, 2016). Different levels of maternal care control GR levels in the hippocampus of the offspring via DNA methylation changes, hence affecting hormonal and behavioral reactivity to stress. Foot shock stress reportedly alters DNA methyltransferase and methylation levels of candidate genes (Miller and Sweatt, 2007). We found that CSDS induces Dnmt3a, a de novo DNA methyltransferase, in NAc of susceptible mice and that Dnmt $3 a$ overexpression in this region increases depression-like behavior, while 
a

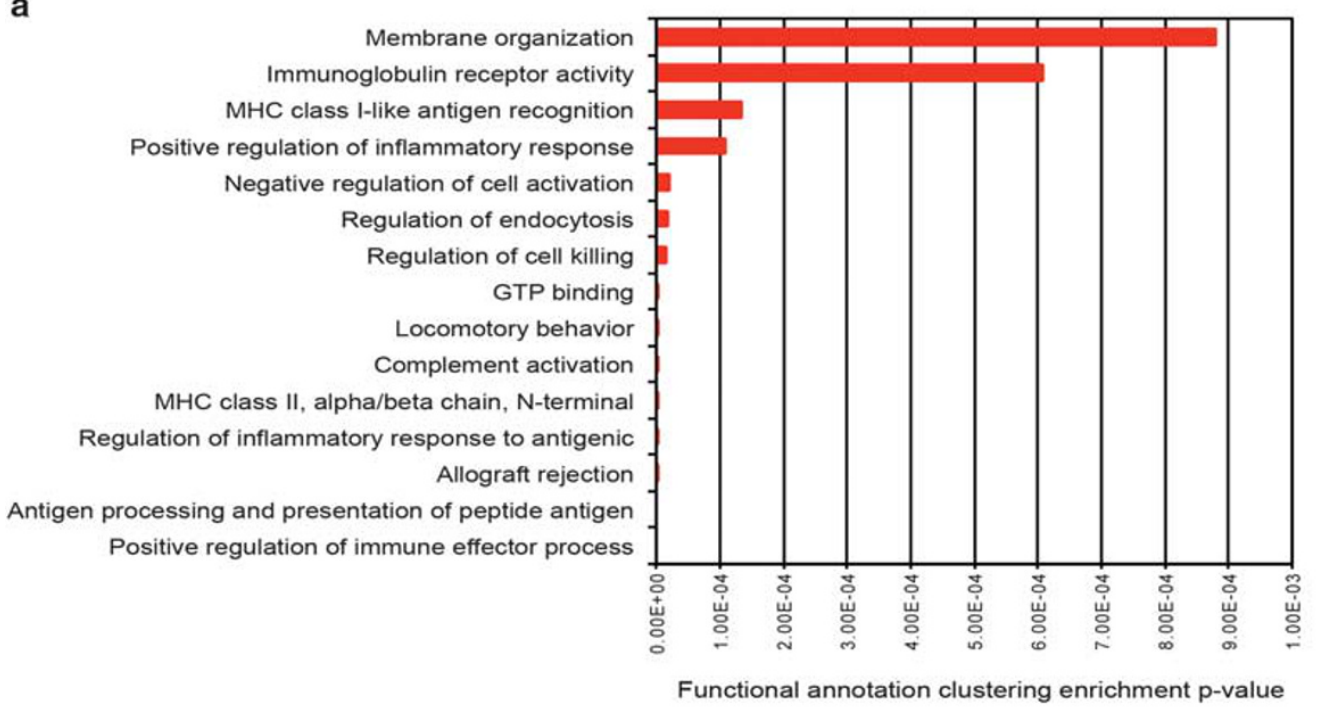

b

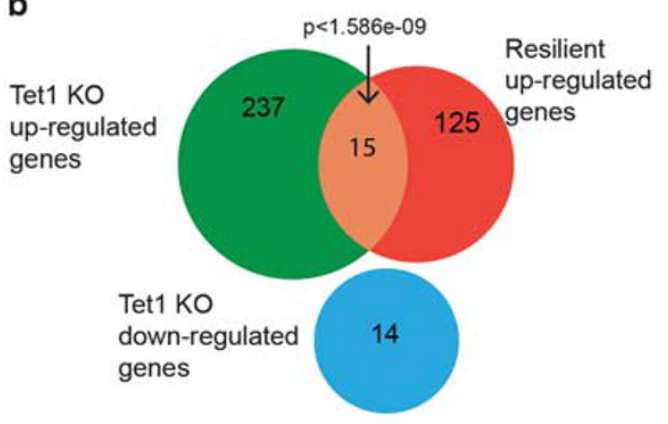

C

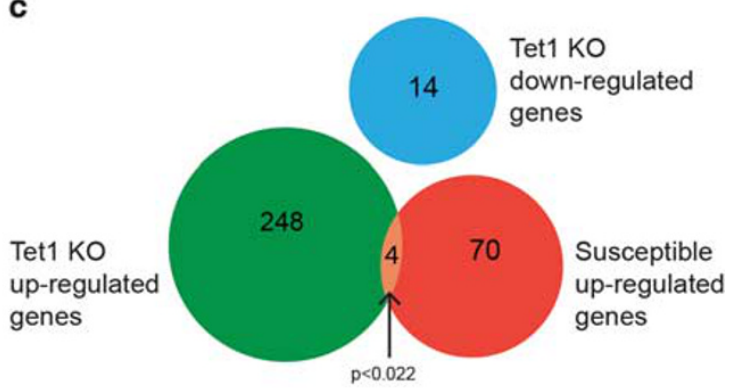

d

\begin{tabular}{cl}
\hline Anxa2 & annexin A2 \\
Bst2 & bone marrow stromal cell antigen 2 \\
Cd74 & CD74 antigen (invariant polypeptide of MHC II antigen-associated) \\
Fgl2 & fibrinogen-like protein 2 \\
Gbp6 & guanylate binding protein 6 \\
$H 2-A a$ & histocompatibility 2, class II antigen A, alpha \\
$H 2-A b 1$ & histocompatibility 2, class II antigen A, beta 1 \\
$H 2-E b 1$ & histocompatibility 2, class II antigen E beta \\
ligp1 & interferon inducible GTPase 1 \\
Lcp2 & lymphocyte cytosolic protein 2 \\
Lyz2 & lysozyme 2 \\
Ngb & neuroglobin \\
PIscr1 & phospholipid scramblase 1 \\
Serping1 & serine (or cysteine) peptidase inhibitor, clade G, member 1 \\
Slc12a7 & solute carrier family 12, member 7 \\
\hline
\end{tabular}

Figure 3 Transcriptome analysis of Tet I KO from NAc. (a) Top I 5 gene ontology enrichment terms and their corresponding P-values. (b) Venn diagrams of differential RNA gene lists reveals significant overlap $(P<1.586 \mathrm{e}-09)$ between Tet $/ \mathrm{KO}$ upregulated genes and genes upregulated in resilient mice 28 days after CSDS. Numbers of genes in each category are noted. (c) Venn diagrams of differential RNA gene lists reveal smaller overlap $(P<0.022)$ between Tet 1 KO upregulated genes and genes upregulated in susceptible mice 28 days after CSDS. (d) List of overlapping genes in panel (b).

intra-NAc infusion of a DNMT inhibitor, RG108, exerts antidepressant-like effects (LaPlant et al, 2010).

The identification of TET enzymes and their products has reinforced the dynamic nature of DNA methylation. However, the role of TETs in brain function, particularly neuropsychiatric disorders, remains largely unknown. Through viral manipulations of TET1, Guo et al (2011) demonstrated that TET1 participates in neuronal activity- induced, active DNA demethylation in the dentate gyrus of adult mice. Using similar approaches, TET1 overexpression was shown to impair memory formation (Kaas et al, 2013). As well, Tet1 mutant mice exhibit abnormal adult hippocampal neurogenesis, long-term depression, and memory extinction (Rudenko et al, 2013; Zhang et al, 2013). Recently, by using viral overexpression and knockdown approaches, we found that TET1 in NAc negatively regulates cocaine 


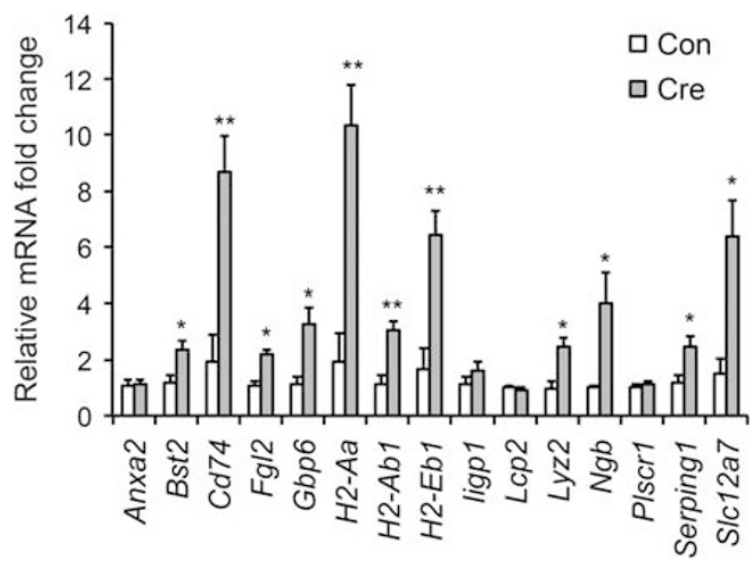

Figure 4 qPCR validation of mRNA transcription change after Tet/ $\mathrm{KO}$. ( $N=5$ for each group. Two-tailed $t$-test, $* P<0.05$, ${ }^{*} * P<0.0$ l.)

reward behavior (Feng et al, 2015). Results of the present study extend these findings by revealing a previously unappreciated role for TET1 in NAc in stress responses.

Our RNAseq data show that the large majority of genes regulated by neuronal Tet1 $\mathrm{KO}$ in NAc of stress-naive mice are immune-related genes. Of note, previous studies have demonstrated a close relationship between alterations in DNA methylation and immune gene expression. For example, conditional $\mathrm{KO}$ of Dnmts in neuroblasts or postmitotic neurons yielded prominent dysregulation of immune gene clusters (Fan et al, 2001; Feng et al, 2010). It would now be important to directly study whether TET1 regulation of immune gene expression is associated with changes in $5 \mathrm{mc}$ or $5 \mathrm{hmc}$ at the affected loci. Our recent study directly linked loss of TET1 with increased $5 \mathrm{hmc}$ and induced gene expression at selective genes (Feng et al, 2015). Although our present RNAseq data confirm the predominant upregulation of genes genome wide upon Tet1 KO, further work is needed to better establish links among TET1, $5 \mathrm{hmc}$, and gene expression. Of note, we did not detect the expression changes for several genes reported previously to be altered upon Tet1 mutation or overexpression (Kaas et al, 2013; Rudenko et al, 2013). Such differences could be attributed to variations in brain region (NAc vs hippocampus), method of gene manipulation (AAV-Cre KO vs AAV overexpression or pan $\mathrm{KO}$ ), or transcriptome profiling approach (RNAseq $v s$ candidate gene analysis).

The significant overlap between genes upregulated in NAc upon Tet1 KO or resilience after CSDS supports an important role of TET1 in stress-related disorders. The fact that most of the overlapping genes fall in immune categories provides further impetus for the importance of immune mechanisms in stress responses. Immune genes have long been implicated in neural development and plasticity and in learning and memory (Huh et al, 2000). Neurons are known to express many genes traditionally characterized in the immune system (Neumann et al, 1997). It is believed that certain immune molecules (eg, MHC I) mediate cellular immunity-like mechanisms in neuronal dendrite pruning and participate in neuropsychiatric diseases (Boulanger and Shatz, 2004; Stephan et al, 2012). Additionally, recent evidence has identified depression-related disruptions in a neuroimmune axis that interfaces between the immune and nervous systems. It is noteworthy that several recent studies have implicated inflammation as a possible cause for at least subtypes of depression and other stress-related disorders (Hodes et al, 2015). Although targeting the neuroimmune axis for depression therapy is still at early stages, our data provide additional supporting evidence for this approach.

In summary, here we identified a novel role of DNA dioxygenase TET1 in stress responses, which offers new insight into both the pathophysiology of depression and the role played by this enzyme in neuronal adaptation. This highlights the importance of DNA epigenetics in the development of stress and other neuropsychiatric disorders and provides a foundation for future improvements in diagnosis and therapy.

\section{FUNDING AND DISCLOSURE}

This work was supported by grants from the National Institute of Mental Health (to EJN), a NARSAD Young Investigator Award (to JF), and the Hope for Depression Research Foundation (HDRF). The authors declare no conflict of interest.

\section{REFERENCES}

Akbarian S (2014). Epigenetic mechanisms in schizophrenia. Dialogues Clin Neurosci 16: 405-417.

Bagot RC, Cates HM, Purushothaman I, Lorsch ZS, Walker DM, Wang J et al (2016). Circuit-wide transcriptional profiling reveals brain region-specific gene networks regulating depression susceptibility. Neuron 90: 969-983.

Bagot RC, Labonte B, Pena CJ, Nestler EJ (2014). Epigenetic signaling in psychiatric disorders: stress and depression. Dialogues Clin Neurosci 16: 281-295.

Berton O, McClung CA, Dileone RJ, Krishnan V, Renthal W, Russo SJ et al (2006). Essential role of BDNF in the mesolimbic dopamine pathway in social defeat stress. Science 311: 864-868.

Boulanger LM, Shatz CJ (2004). Immune signalling in neural development, synaptic plasticity and disease. Nat Rev 5: 521-531.

Cheng Y, Bernstein A, Chen D, Jin P (2015). 5-Hydroxymethylcytosine: a new player in brain disorders? Exp Neurol 268: 3-9.

Dawlaty MM, Ganz K, Powell BE, Hu YC, Markoulaki S, Cheng AW et al (2011). Tet1 is dispensable for maintaining pluripotency and its loss is compatible with embryonic and postnatal development. Cell Stem Cell 9: 166-175.

Dias C, Feng J, Sun H, Shao NY, Mazei-Robison MS, Damez-Werno D et al (2014). beta-catenin mediates stress resilience through Dicer1/ microRNA regulation. Nature 516: 51-55.

Fan G, Beard C, Chen RZ, Csankovszki G, Sun Y, Siniaia M et al (2001). DNA hypomethylation perturbs the function and survival of CNS neurons in postnatal animals. J Neurosci 21: 788-797.

Feng J, Shao N, Szulwach KE, Vialou V, Huynh J, Zhong C et al (2015). Role of Tet1 and 5-hydroxymethylcytosine in cocaine action. Nat Neurosci 18: 536-544.

Feng J, Wilkinson M, Liu X, Purushothaman I, Ferguson D, Vialou V et al (2014). Chronic cocaine-regulated epigenomic changes in mouse nucleus accumbens. Genome Biol 15: R65.

Feng J, Zhou Y, Campbell SL, Le T, Li E, Sweatt JD et al (2010). Dnmt1 and Dnmt3a maintain DNA methylation and regulate synaptic function in adult forebrain neurons. Nat Neurosci 13: 423-430.

Golden SA, Covington HE 3rd, Berton O, Russo SJ (2011). A standardized protocol for repeated social defeat stress in mice. Nat Protoc 6: 1183-1191. 
Guidotti A, Dong E, Gavin DP, Veldic M, Zhao W, Bhaumik DK et al (2013). DNA methylation/demethylation network expression in psychotic patients with a history of alcohol abuse. Alcohol Clin Exp Res 37: 417-424.

Guo JU, Su Y, Zhong C, Ming GL, Song H (2011). Hydroxylation of 5 -methylcytosine by TET1 promotes active DNA demethylation in the adult brain. Cell 145: 423-434.

Hodes GE, Kana V, Menard C, Merad M, Russo SJ (2015). Neuroimmune mechanisms of depression. Nat Neurosci 18: 1386-1393.

Huang, da W, Sherman BT, Lempicki RA (2009). Systematic and integrative analysis of large gene lists using DAVID bioinformatics resources. Nat Protoc 4: 44-57.

Huh GS, Boulanger LM, Du H, Riquelme PA, Brotz TM, Shatz CJ (2000). Functional requirement for class I MHC in CNS development and plasticity. Science 290: 2155-2159.

Hyman S (2014). Mental health: depression needs large humangenetics studies. Nature 515: 189-191.

Jaenisch R, Bird A (2003). Epigenetic regulation of gene expression: how the genome integrates intrinsic and environmental signals. Nat Genet 33: 245-254.

Kaas GA, Zhong C, Eason DE, Ross DL, Vachhani RV, Ming GL et al (2013). TET1 controls CNS 5-methylcytosine hydroxylation, active DNA demethylation, gene transcription, and memory formation. Neuron 79: 1086-1093.

Kriaucionis S, Heintz N (2009). The nuclear DNA base 5hydroxymethylcytosine is present in Purkinje neurons and the brain. Science 324: 929-930.

Krishnan V, Han MH, Graham DL, Berton O, Renthal W, Russo SJ et al (2007). Molecular adaptations underlying susceptibility and resistance to social defeat in brain reward regions. Cell 131: 391-404.

Krishnan V, Nestler EJ (2008). The molecular neurobiology of depression. Nature 455: 894-902.

LaPlant Q, Vialou V, Covington HE 3rd, Dumitriu D, Feng J, Warren BL et al (2010). Dnmt3a regulates emotional behavior and spine plasticity in the nucleus accumbens. Nat Neurosci 13: 1137-1143.

Law CW, Chen Y, Shi W, Smyth GK (2014). voom: Precision weights unlock linear model analysis tools for RNA-seq read counts. Genome Biol 15: R29.

Li X, Wei W, Zhao QY, Widagdo J, Baker-Andresen D, Flavell CR et al (2014). Neocortical Tet3-mediated accumulation of 5-hydroxymethylcytosine promotes rapid behavioral adaptation. Proc Natl Acad Sci USA 111: 7120-7125.

Miller CA, Sweatt JD (2007). Covalent modification of DNA regulates memory formation. Neuron 53: 857-869.

Neumann H, Schmidt H, Cavalie A, Jenne D, Wekerle H (1997). Major histocompatibility complex (MHC) class I gene expression in single neurons of the central nervous system: differential regulation by interferon (IFN)-gamma and tumor necrosis factor (TNF)-alpha. J Exp Med 185: 305-316.

Rudenko A, Dawlaty MM, Seo J, Cheng AW, Meng J, Le T et al (2013). Tet1 is critical for neuronal activity-regulated gene expression and memory extinction. Neuron 79: 1109-1122.

Russo SJ, Nestler EJ (2013). The brain reward circuitry in mood disorders. Nat Rev 14: 609-625.

Stephan AH, Barres BA, Stevens B (2012). The complement system: an unexpected role in synaptic pruning during development and disease. Annu Rev Neurosci 35: 369-389.

Sun H, Damez-Werno DM, Scobie KN, Shao NY, Dias C, Rabkin J et al (2015). ACF chromatin-remodeling complex mediates stress-induced depressive-like behavior. Nat Med 21: $1146-1153$.

Szulwach KE, Li X, Li Y, Song CX, Wu H, Dai Q et al (2011). 5-hmC-mediated epigenetic dynamics during postnatal neurodevelopment and aging. Nat Neurosci 14: 1607-1616.

Tahiliani M, Koh KP, Shen Y, Pastor WA, Bandukwala H, Brudno Y et al (2009). Conversion of 5-methylcytosine to 5-hydroxymethylcytosine in mammalian DNA by MLL partner TET1. Science 324: 930-935.

Turecki G, Meaney MJ (2016). Effects of the social environment and stress on glucocorticoid receptor gene methylation: a systematic review. Biol Psychiatry 79: 87-96.

Vialou V, Feng J, Robison AJ, Nestler EJ (2013). Epigenetic mechanisms of depression and antidepressant action. Annu Rev Pharmacol Toxicol 53: 59-87.

Yu H, Su Y, Shin J, Zhong C, Guo JU, Weng YL et al (2015). Tet3 regulates synaptic transmission and homeostatic plasticity via DNA oxidation and repair. Nat Neurosci 18: 836-843.

Zhang RR, Cui QY, Murai K, Lim YC, Smith ZD, Jin S et al (2013). Tet1 regulates adult hippocampal neurogenesis and cognition. Cell Stem Cell 13: 237-245.

Zhao Z, Chen L, Dawlaty MM, Pan F, Weeks O, Zhou Y et al (2015). Combined loss of Tet 1 and Tet 2 promotes B cell, but not myeloid malignancies, in mice. Cell Rep 13: 1692-1704. 